\title{
Fluctuation Microscopy Analysis of Amorphous Silicon Models
}

\author{
J. M. Gibson* \\ Northeastern University, Department of Physics, Boston MA 02115 \\ $\&$ \\ M.M.J. Treacy \\ Arizona State University, Department of Physics, Tempe AZ 85287
}

January 3, 2017

\begin{abstract}
Using computer-generated models we discuss the use of fluctuation electron microscopy (FEM) to identify the structure of amorphous silicon. We show that a combination of variable resolution FEM to measure the correlation length, with correlograph analysis to obtain the structural motif, can pin down structural correlations. We introduce the method of correlograph variance as a promising means of independently measuring the volume fraction of a paracrystalline composite. From comparisons with published data, we affirm that only a composite material of paracrystalline and continuous random network that is substantially paracrystalline could explain the existing experimental data, and point the way to more precise measurements on amorphous semiconductors. The results are of general interest for other classes of disordered materials.
\end{abstract}

\section{Introduction}

While FEM has been repeatedly confirmed as a powerful "fingerprint" for medium-range order in amorphous materials, it remains difficult to simply interpret the results. For example, if we focus on the archetypal amorphous silicon, and the paracrystalline [1] versus continuous random network (CRN) [2] models, several questions have been difficult to separate. The paracrystalline model assumes a conglomerate of small crystallites that are imperfect. In fact in the classic model where the amorphous material is a compact of paracrystallites the degree of order decays as a function of distance from any atom [1]. Real models of nanocrystals have distortions that increase near the grain boundaries and the decay length is approximately the same as the length scale of the grain size. However,

* current address FAMU/FSU Joint College of Engineering, 225 Pottsdamer Street, Tallahassee, FL 32310

(C) 2017. This manuscript version is made available under the Elsevier user license http://www.elsevier.com/open-access/userlicense/1.0/ 
it is also possible that the real material is a composite of paracrystals embedded in a random network. In that case key parameters would be the grain-size or correlation length and the volume fraction of paracrystalline material. Using the "ansatz" model proposed by Gibson et al. [3], several authors have had consistent results in measuring the correlation length from the dependence of the normalized intensity variance on the probe size (also known as "variable resolution microscopy") e.g. Bogle et al. [4]. There has been controversy about the volume fraction. Some authors not using FEM have claimed that, while ordered regions may be present, they are only in trace concentrations (e.g. Wang et al. [5]). It has thus become important to develop the FEM technique so that it can separately determine; 1) the structure of ordered regions; 2) the size or correlation length of the order; and 3) the volume fraction of ordered regions. Based on a variety of previous works, and some new ideas discussed here, we feel confident that these should be independently measurable by experiment. We support our claim in this paper through simulations on a variety of models for amorphous silicon. We expand on these three issues next

1) The "crystal structure" 1 of ordered regions.

From the pioneering work of Kam [6] it is known that the correlation function,

$$
C=\frac{\left\langle I\left(\underline{k}_{1}\right) I\left(\underline{k}_{2}\right)\right\rangle}{\left\langle I\left(\underline{k}_{1}\right)\right\rangle\left\langle I\left(\underline{k}_{2}\right)\right\rangle}
$$

recovers the single-particle correlation function, assuming a distribution of randomly-oriented ordered clusters. The "correlograph", introduced for electron microscopy by Gibson and Treacy [7], based on ideas of Cheng et al. [8], is the azimuthal autocorrelation function of $C$. We previously noted, in contrast to the related x-ray approach of Wochner et al. [9], that the ensemble-averaged $C$ was essential to reveal single particle correlation functions. Probing individual images before averaging in a correlograph can be interesting, but coincidental and unreal symmetries can be displayed due to particle overlap. These are averaged out in the correlograph [7]. The correlograph is effective for directly revealing the structure of the average unit, such as the structure of paracrystals. We have shown, and elaborate here, that the paracrystalline model, amongst those models currently proposed, is the only model that comes close to fitting the experimental correlograph.

The FEM normalized intensity variance plot can be used to deduce the crystal structure through simulations, but this is indirect and places a premium on the construction of comprehensive families of physically realistic structures. Experimentally-constrained Monte Carlo approaches using FEM variance appear to be effective, requiring very little a priori model building $[10,11,12]$. In all cases the paracrystalline structure was found to be a good fit to data and, in at least one case, it was the only acceptable fit [11]. In one study [10], a CRN model that contained voids was found to fit also (vide infra). In another study [12] a complication arose because the procedure for obtaining the normalized variance was incon-

\footnotetext{
${ }^{1}$ We use quotes around "crystal structure" because there is no long-range order constraint, and non-periodic packing symmetries, such as dodecahedral, are possible.
} 
sistent between experiment and modeling (vide infra). However, we will see that FEM data obtained with only one probe size, without knowledge of scaling, is not sufficiently constraining. The fluctuation map [3] (i.e., the 3 -dimensional plot of normalized variance against scattering vector and probe size) is far more constraining, but has not yet been used to our knowledge in experimentally-constrained energy-minimization. Furthermore, the correlograph contains much more information, about symmetry for example, and should also be considered as an experimental constraint for minimization.

2) The "crystal" size, or correlation, length

Given the existence of ordered regions, measurement of their size or correlation length $\Lambda$ is among the most reliable and widely used experimental methods. Gibson et al. [3] proposed an "ansatz" model for the measurement of the correlation length, the validity of which has repeatedly been demonstrated experimentally and by simulation e.g. [4, 13]. The ansatz model approximates the simulation of variance by assuming that the contributions arising from probe size (beam convergence) and scattering vector are decoupled - literally, a separation of variables where we declare that $V\left(r_{p}, k\right) \equiv R\left(r_{p}\right) K(k)$. This has been shown to be an excellent approximation when the probe size is larger than the correlation length, i.e. $r_{p} \gg \Lambda$. In this paper we also demonstrate that an even more direct measurement of the correlation length can be obtained by identifying the probe size that yields the maximum overall variance. This occurs at $r_{p} \approx \Lambda$. For the case of static amorphous silicon models, we find that experimentally observed fluctuation maps are consistent only with a paracrystalline structure - neither the CRN nor the CRN with voids show the characteristics of medium-range order. Nor do they show a peak and decay in the variance versus probe size. Of course this does not rule out the possibility of a composite paracrystalline and CRN-type model, and that question is addressed next.

3) Volume fraction of ordered regions

This has proven to be the most difficult to measure reliably. While theoretically there is a strong correlation between the fraction of order and the magnitude of the variance peaks, there are suspicions that "decoherence" effects strongly reduce these experimental variance amplitudes in an as-yet unpredictable way $[14,15]$. The structure details would also affect the variance peak heights so that questions 1 and 3 are difficult to decouple. Several approaches have been suggested. Bogle et al.[4] observed a correlation between the relative heights of the first and second variance peak, at least for $\mathrm{Si}$, but this depends also on the probe size. Yi et al. [16] proposed detailed fitting of peak heights and probe size, based on a reasonably sophisticated dispersed crystal model of a composite, but this appears vulnerable to details of the structure (e.g. faulting and intergrowths).

An intriguing, and highly informative, metric to examine is the correlograph. Gibson et al. [7] showed that the characteristics of correlographs were affected significantly by volume fraction, and used that sensitivity to estimate a paracrystalline volume fraction of greater than $50 \%$ in a 
typical sputtered amorphous Si sample. On the other hand, Raman data has been used to suggest a much smaller volume fraction [5]. Cheng et al. [8] proposed diffraction intensity cross-correlations (covariance) as a more informative measure than the simple variance, which measures the intensity autocorrelation. Li et al. [17] went further and proposed crosscorrelations as an approach to study volume fraction. Below we suggest that the variance of the correlograph looks like a powerful and independent method to determine volume fraction on the assumption (which can be verified by conventional diffraction) that there is a random orientation distribution of paracrystals.

From our modeling of amorphous silicon it seems clear that the paracrystalline structure (possibly diluted with CRN) is the only model that can explain FEM data, including correlographs, experimentally observed in amorphous silicon. This assertion is also consistent with experimentallyconstrained energy minimization [11]. The 2-body function is not discriminating enough to distinguish these models, as has already been clearly demonstrated $[11,12]$. However, detailed comparison with fresh experiments, including measurements of the correlograph variance, is desired to make more precise conclusions.

\section{Theoretical and simulation background}

Simulations were carried out including the curvature of the Ewald sphere under the kinematical approximation. To deal with small model sizes simulations were carried out at $20 \mathrm{kV}$ accelerating voltage, which replicates the conditions of multiple scattering at $100-200 \mathrm{kV}$ in a typical thickness of 10-20 nm. For comparison, full multi-slice calculations and weak-phase object calculations were also made using the same Mathematica-based software package written by the first author, JMG. In general we saw reduced peak heights, but no major changes in thin samples arising from multiple scattering, and little accelerating voltage dependence apart from Friedel's-law issues arising from the curvature of the Ewald sphere. Models up to $20 \mathrm{~nm}$ in size were simulated. In order to expand the size of smaller models, these were rotated through 90 degrees, shifted and assembled into a larger cube, from which cubes of size $\sqrt{3}$ times the original model size could be carved after random re-orientation. About 1000 image/diffraction calculations were carried out for each model and for each probe size.

Variance is calculated by the technique listed as number 4 ("Annular mean of variance image") by Daulton et al. [18],

$$
V=\frac{\left\langle\left\langle I^{2}(\underline{k})\right\rangle_{r}\right\rangle_{\phi}}{\left\langle\langle I(\underline{k})\rangle_{r}^{2}\right\rangle_{\phi}},
$$

where \langle\rangle$_{\phi}$ is the average over the azimuthal angle, $\phi$, and the average, \langle\rangle$_{r}$, is over the ensemble (that is, over different probe locations, $\mathbf{r}$ ).

This method produces much higher variance overall than the first method enumerated by Daulton ("Normalized variance of the annular mean"), which evaluates the angular average of $I(\underline{k})^{2}$ before averaging 
over the ensemble, and so method 4 is much preferable to method 1 . We should note here that method 1 was initially used in hollow-cone dark-field in the earliest FEM work because the angular averaging automatically occurred. The angular averaging was originally seen as an advantage as it provided a way to adjust the illumination spatial coherence, and the method was originally referred to as variable coherence microscopy [19]. However, the hollow-cone approach has been generally superseded by data from either single-tilt-illumination dark-field or STEM nanodiffraction, in which the angular averaging is not automatic.

The Debye method of calculating diffraction intensity variance from models (McBride et al. [20]) automatically invokes angular averaging, and so its results should not be compared with those from other methods. The Debye method loses a significant amount of variance sensitivity, and in general is not attractive for FEM simulations. Unfortunately, it seems that Peterson et al. [12] not only used the Debye method, but may have compared it with experimental data that was obtained by Daulton's method 4 . This discrepancy probably accounts for the differing conclusions reached by Peterson et al. [12] and Borisenko et al. [11]. In general, we recommend the use of the more sensitive experimental method \#4 where possible, and not using the Debye simulation approach because it attenuates variance. Nevertheless, the Debye method (\#1) is still often used, since this was the original approach used with hollow-cone illumination in TEM. Since most data is now collected in the nanodiffraction mode with STEM, alternate analyses can be easily compared from the same experimental dataset.

\section{$3 \quad$ Results}

\subsection{Continuous Random Network Models}

We examined three continuous random network (CRN) models, and found similar results for each. None of the CRN models exhibit significant topographically crystalline regions as revealed in figure 1(a) for the Wooten, Winer, Weaire model [21], which highlights in red those regions where there is a cluster of twenty or more atoms with dihedral angles within 4 degrees of the staggered diamond configuration. Systematically, the static CRN model cannot fit the fluctuation maps. It is possible to obtain peaks in the variance map at a particular probe size, nevertheless the magnitude of the peaks is an order of magnitude smaller than for the smallest grain paracrystalline models (1 $\mathrm{nm}$ grain size). A fluctuation map for the Barkema [22] 4096-atom model is shown in figure 2(a), and reveals no decay region or peaks at the characteristic $0.31 \AA^{-1}$ and $0.52 \AA^{-1}$ positions that are observed experimentally.

The correlograph for a CRN is shown in figure 3(a). While the $111 \mathrm{ring}$ at $0.31 \AA^{-1}$ shows faint correlations associated with the tetrahedral geometry, there is no evidence for pronounced correlations at higher scattering vector, $k$. Interestingly, correlographs are closely related to Patterson maps of the structure [6].

Recently Treacy et al. has suggested that "decoherence" effects could produce peaks in the normalized variance of a CRN [14]. We are able to re- 
produce this effect by introducing an anomalously high thermal vibration amplitude. However, this thermal amplitude would be a equivalent to a temperature far in excess of the melting point. Further, our calculations show that under such conditions the diffraction pattern $I(k)$ is significantly smeared, inconsistent with experiment. As pointed out by Treacy et al., the displacement decoherence is probably not of Debye-Waller type in origin (i.e., individual atomic displacements), but probably related to flexural tilts of local regions, induced by the beam, which would give rise to the intensity twinkling that is observed during collection of diffraction patterns. Curiously, the correlograph from a CRN that has been decohered by a large amplitude Debye-Waller-type thermal motion does not look significantly different from that of the static CRN. The issue of decoherence, and how to simulate it quickly, is an important one to resolve for quantitative FEM analysis.

\subsection{Paracrystalline Models}

Paracrystalline models are made in the computer by generating randomly oriented crystallites of predetermined size and structure, in most cases embedding these in a continuous random network matrix, and most often minimizing the configurational energy. One paracrystalline structure was generated as a best fit to experimental data in an experimentallyconstrained Monte Carlo approach [11]. An example of a Nakhmanson paracystalline model with four grains in a 4096-atom cube is shown in fig 1(b) [24]. We examine particularly those structures produced by Voronoi tesselation of a cubic volume [25]. The underlying crystal structures of the grains studied here were diamond silicon. These models show similar features depending on the grain size and the volume fraction of crystalline material, with a small variation in diffraction intensity. We examined a number of paracrystalline models and observed some cautions. Early models had a very small number of crystalline grains (e.g four in the Keblinski [23] and Nakhmanson [24] models), and in the case of the Nakhmanson model there was a strong coincidental alignment correlation between a pair of grains. In such cases artificial correlations can be superimposed due to the granular spacing. The largest paracrystalline models that we have examined were produced by Treacy using Voronoi tesselation [25]. These models do not show significant grain-correlation artifacts. A typical model of size 75 Åcontains $\sim 100$ grains. The larger Treacy models were not relaxed [25], and we have briefly explored the effects of relaxation using the LAMMPS molecular dynamics code (http://lammps.sandia.gov) [26]. The effect of relaxation is to reduce the peak intensities but not otherwise to create dramatic differences.

The fluctuation map from a typical paracrystalline model (fig 2(b)) shows the resolution dependence observed experimentally, with a decaying variance as probe size increases that can be used to estimate the correlation length. From these simulations we can measure the correlation length by the ansatz method [3] and by looking at the probe size that causes the highest variance. The results are given in Table I, revealing that both methods can estimate the grain size with reasonable accuracy. 


\subsection{Continuous Random Network with Voids}

Biswas et al. [10] confirmed the inability of a static CRN structure to fit FEM data, but were able to fit data with paracrystalline models (to within an adjustable multiplicative parameter that compensated for variance suppression by decoherence). However, they also reported that a CRN model with a high void density could fit the experimental data in a "reverse Monte Carlo," experimentally-constrained energy minimization. While the void density (typically $\sim 20 \%$ ) is much higher than seen experimentally, the result is important in that it is the only non-paracrystalline model that has fit the data. We have examined several CRN-void models. We find that, while a variance profile at a single probe size can give an acceptable fit to experiment, fluctuation maps that sample many probe sizes do not show the characteristic probe size dependence observed experimentally, and associated with real interatomic correlations (see figure 2 (c), for example). The height of the variance peaks is also substantially lower than for paracrystalline models (with static models). In addition, the correlograph (fig. 3 (c)) does not fit the data and is not easily distinguishable from the CRN (fig. 3(a)). It seems possible that the fit of this model is an artifact of fitting a CRN with looser constraints (due to voids) to FEM data, especially without considering the absolute magnitude of the variance. There is no evidence for long-range correlations in the atomic positions such as in figure 1 (c). It should be noted that published models reviewed by the author have used spherical voids, whereas experimental evidence suggests that voids in amorphous silicon are anisotropic [27]. This aspect that might be interesting to explore further with modeling.

\subsection{Volume Fraction for Paracrystalline/CRN Com- posites}

Li et al. [17] showed that the covariance of two independent scattering vectors gives insight into the volume fraction of paracrystallites, depending on the characteristic crystallite size. We propose a related, but more direct, method for obtaining the volume fraction by examining the variance of the correlograph itself. This is obtained from an ensemble of single-point correlographs in the expected way. It can be thought of as a correlation function that is higher than fourth-order, since the correlograph itself is already a fourth-order function of atom position. It seems clear how this would work. If the density of paracrystals is very low, the probability of more than one in each probed region of the ensemble would be small, and we would never see more than one excited set of Bragg reflections at once. For example, if we look at the $220 \mathrm{ring}$ at $0.52 \AA^{-1}$, we might see more than one 220 reflection but never at angles different from the 90 degrees or 60 degrees that are characteristic of [100] or [111] projections of a small crystallite. If more than one 220 reflection is seen in any one diffraction pattern at angles different from this, it would imply more than one crystal. And of course the probability of more than one crystal would increase with the density. A simple model indicates that the variance of the intensity at regions away from the characteristic angles would depend linearly on the crystallite density, when appropriately normalized 
This is revealed in figure 5, where the variance of correlographs for three different concentrations of paracrystals diluted with CRN are show. The graph in fig. 5(d) reveals the background-subtracted variance away from zone axis azimuthal correlations for the 220 reflection $\left(0.52 \AA^{-1}\right)$, i.e. multiples of 60 and 90 degrees. The background-subtracted variance is normalized to the 180-degree Friedel peak, which scales with the density of reflections. While we still need to examine how to optimally normalize this eight-order information with respect to experimental data, the method seems to point at an independent reliable approach to measuring crystallite density. The only assumption required is that the crystallites have no orientational correlation, although this could likely also be verified by examining the data for correlated reflection angles.

\section{Conclusion}

Examination of several competing models of amorphous silicon using fluctuation microscopy reveals that a combination of FEM techniques should be able to distinguish any paracrystalline motif, structure and size, and the volume density of paracrystalline material in a composite with continuous random network.

We introduced some new results - showing that a CRN-with-voids model does not fit data well, despite previous assertions [10], and proposing a new approach to measuring the volume fraction by studying the variance of the correlograph. We also resolved inconsistencies in the conclusions of Petersen et al. [12] and Borisenko et al. [11] by identifying the ineffectiveness of the Debye approximation [20] for the simulations of FEM experimental variance. As a result it seems consistent that only the paracrystalline models, diluted with $\mathrm{CRN}$, can explain FEM data.

While modeling and inspection of data, initially used widely to interpret FEM data, may work well, Experimentally-Constrained Minimization is the most rigorous current approach to data interpretation. However, our simulations show that such data must include a richer collection of FEM data, and not just "variable coherence" data with no absolute measurement. Instead, the dependence of variable coherence data on probe size (i.e. the fluctuation map [3]), and possibly the correlograph and correlograph variance, would be far more constraining in reaching conclusions about the exact structure. Further, our simulations indicate that the correlograph is not as adversely affected by decoherence as is the normalized variance. This suggests that the correlograph may be a more robust data constraint for model-optimization procedures.

Nevertheless, the fact that diffraction simulations on static structures grossly overestimate the variance compared with experimental data, is an important issue that needs to be resolved computationally. Displacement decoherence appears to be the primary culprit, where the electron beam disrupts the sample during exposure [14]. The diffraction pattern twinkles rapidly during the exposure because the beam induces local motion. In addition to beam damage (atoms being knocked off sites), long-range flexural modes can tilt significant volumes of the material, causing the diffraction intensity from quite large volumes to fluctuate. Twinkling at 
time scales of $10 \mathrm{~ms}$ and longer can be observed directly by watching the diffraction pattern under a stationary probe. The time-averaged outcome significantly suppresses the speckle intensity, which is what FEM measures. Decoherence is further exacerbated by multiple scattering in thicker samples, pointing to the need for multi-slice simulations for thicker samples. In this study, we do not include the effects of decoherence because its simulation is computationally demanding. Its omission from this study does not invalidate the conclusions we draw about the merits of correlographs, but it will need to be addressed for reliable quantitative studies.

Although FEM is still not a fully quantitative method, becasue of the complexities of simulating the electron scattering from a sample moving under the electron beam, estimates of crystallite density, size and structure can still be inferred remarkably reliably by the analysis described here.

\section{Acknowledgements}

The authors are grateful to Northeastern University for financial support during a sabbatical for JMG. MMJT was supported by the U.S. Department of Energy, Office of Basic Energy Sciences, Division of Materials Sciences and Engineering under Award DE-PS02-09ER09-01.

\section{Figures}

Table I: From calculations on Voronoi paracrystalline models with different grain sizes [25], we calculate the grain size from the position of the peaks in the variance (columns 1 and 2), and from the ansatz model [3] in which case the grain size is given by multiplying the correlation length by $\sqrt{10}[25]$.

Figure 1: Typical ball and stick pictures of representative models used in the FEM calculations: a) Continuous Random Network [21]; b) Paracrystalline model [24]; c) Voided continuous random network model [10]. In each case clusters containing $>19$ interconnected atoms where the dihedral angle is $180 \pm 4$ degrees are highlighted in red to reveal crystallinelike atomic correlations.

Figure 2: Fluctuation maps for: a) Continuous Random Network (Barkema [22]); b) Paracrystalline model with 4 grains [24]; c) Voided continuous random network model [10].

Figure 3: Correlographs for: a) Continuous Random Network [22]; b) Paracrystalline model [24]; c) Continuous random network model with voids [10].

Figure 4: The dependence of the correlograph variance on paracrystal size in the voronoi model with $75 \AA$ model size. In this case all cells in the model were filled with diamond crystals.

Figure 5: The variance of the correlograph for three different $15 \AA$ paracrystal composites in the Voronoi model [25] with densities a) $25 \%$ b) $50 \%$ and c) $100 \%$. The non-crystalline cells in these $75 \AA$ models are filled with CRN. 


\section{References}

[1] J. M. Cowley, in "Diffraction Studies on Non-Crystalline Materials," 13, Stud. Phys. Theor. Chem., Hungarian Academy of Sciences, Budapest, 847-891 (1981)

[2] D. E. Polk, J. Non-Cryst. Sol. 5, 365 (1971)

[3] J. M. Gibson, M. M. J. Treacy and P. M. Voyles, Ultramicroscopy 83, 169 (2000)

[4] S. N. Bogle, L. N. Nittala, R. D. Twesten, P. M. Voyles and J. R. Abelson, Ultramic. 110, 1273 (2010)

[5] X. D. Wang, B. Chen, H. F. Wang, B. Chen, S. J. liu, Z-X Cui, B. Li, J-B Wang, S-M Wang and Y-P Li, J. Appl. Cryst. 48, 1011 (2015)

[6] Z. Kam, Macromolecules, 10, 927 (1977)

[7] J. M. Gibson, M. M. J. Treacy, T. Sun and N. J. Zaluzec, Physical Review Letters 105, 125504 (2010)

[8] J. Y. Cheng, M. M. J. Treacy, P. J. Keblinksi and J. M. Gibson, J. Appl. Phys. 95, 7779 (2004)

[9] P. Wochner, C. Gutt, T. Autenrieth, T. Demmer, V. Bugaev, A. D. Ortiz, A. Duri, F. Zontone, G. Grubel and H. Dosch, Proc. Natl. Acad. Sci. USA 106, 11511 (2009)

[10] P. Biswas, R. Atta-Fynn, S. Chakraborty and D. A. Drabold, J. Phys. Cond. Matter 19, 455202 (2007)

[11] M. M. J. Treacy and K. B. Borisenko, Science 335, 950 (2012)

[12] T. C. Petersen, G. Opletal, A. C. Y. Liu and S. P. Russo, Molecular Simulation 15th Sept 2015

[13] P. M. Voyles and D. A. Muller, Ultramic. 93, 147 (2002)

[14] A. Rezikyan, Z. J. Jibben, B. A. Rock, G. Zhao, F. A. M. Koeck, R. F. Nemanich and M. M. J. Treacy, Micros. and Microanal., 21, 1455 (2015)

[15] D. Van Dyck, I. Lobato, F.-R. Chen and C. Kisielowski, Micron 68, $158(2014)$

[16] F. Yi and P. M. Voyles, Ultramic. 122, 37 (2012)

[17] T. T. Li, K. Darmawikarta, and J. R. Abelson, Ultramic. 133, 95 (2013)

[18] T. L. Daulton, K. S. Bondi and K. F. Kelton, Ultramicroscopy 110, 1279 (2010)

[19] M. M. J. Treacy and J. M. Gibson, Acta Cryst. A 52, 212 (1996)

[20] W. McBride and D. J. H. Cockayne J. Non Cryst. Solids 318, 233 (2003)

[21] F. Wooten, K. Winer and D. Weaire, Phys. Rev. Lett. 54, 1392 (1985)

[22] G. T. Barkema and N. Mousseau, Phys. Rev. B 62, 4985 (2000)

[23] P. Keblinski, S. R. Phillpot, D. Wolf and H. Gleiter, Acta Materiala 45, 987 (1997) 
[24] S. M. Nakhmanson, P. M. Voyles, N. Mousseau, G. T. Barkema and D. A. Drabold, Phys. Rev. B 63, 235207 (2001)

[25] M. M. J. Treacy and J. M. Gibson, Microsc Microanal 18, 241 (2012)

[26] S. J. Plimpton, J. Comp. Phys. 117, 1 (1995)

[27] B. Haberl , S.N. Bogle, T. Li, I. McKerracher, S. Ruffell, P. Munroe, J.S. WIlliams, J.R. Abelson and J.R. Bradby, J. Appl. Phys. 096104 (2011) 


\section{Table I}

\begin{tabular}{l|l|l|l|l|}
\hline $\begin{array}{l}\text { Grain } \\
\text { Size }\end{array}$ & $\begin{array}{l}111 \text { Peak } \\
(\AA)\end{array}$ & $\begin{array}{l}220 \text { Peak } \\
(\AA)\end{array}$ & $\begin{array}{l}\text { Ansatz } \\
111(\AA)\end{array}$ & $\begin{array}{l}\text { Ansatz } \\
220(\AA)\end{array}$ \\
\hline $\begin{array}{l}10 \AA \\
\text { Grain }\end{array}$ & 11.1 & 12.1 & $11.4 \pm 1.7$ & $9.5 \pm 1.3$ \\
\hline $\begin{array}{l}15 \AA \\
\text { Grain }\end{array}$ & 13.2 & 13.4 & $12 \pm 1.9$ & $13.3 \pm 0.8$ \\
\hline $\begin{array}{l}20 \AA \AA \\
\text { Grain }\end{array}$ & 17.1 & 16.4 & $16.8 \pm 2$ & $17.4 \pm 1$ \\
\hline
\end{tabular}




\section{Figure 1}

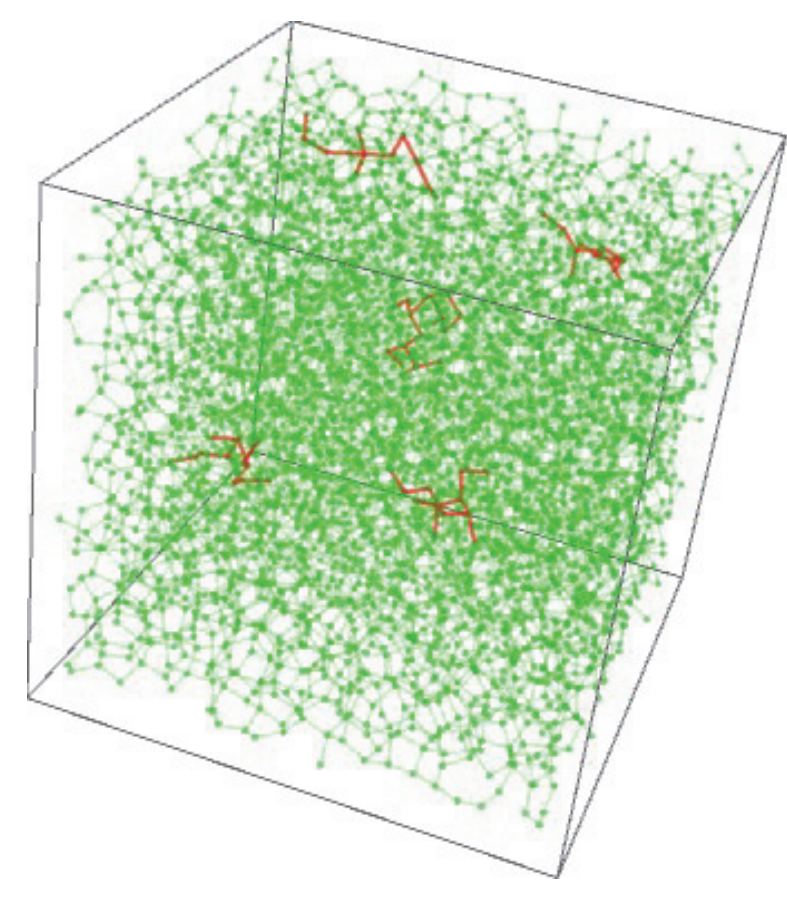

a) CRN (Wooten)

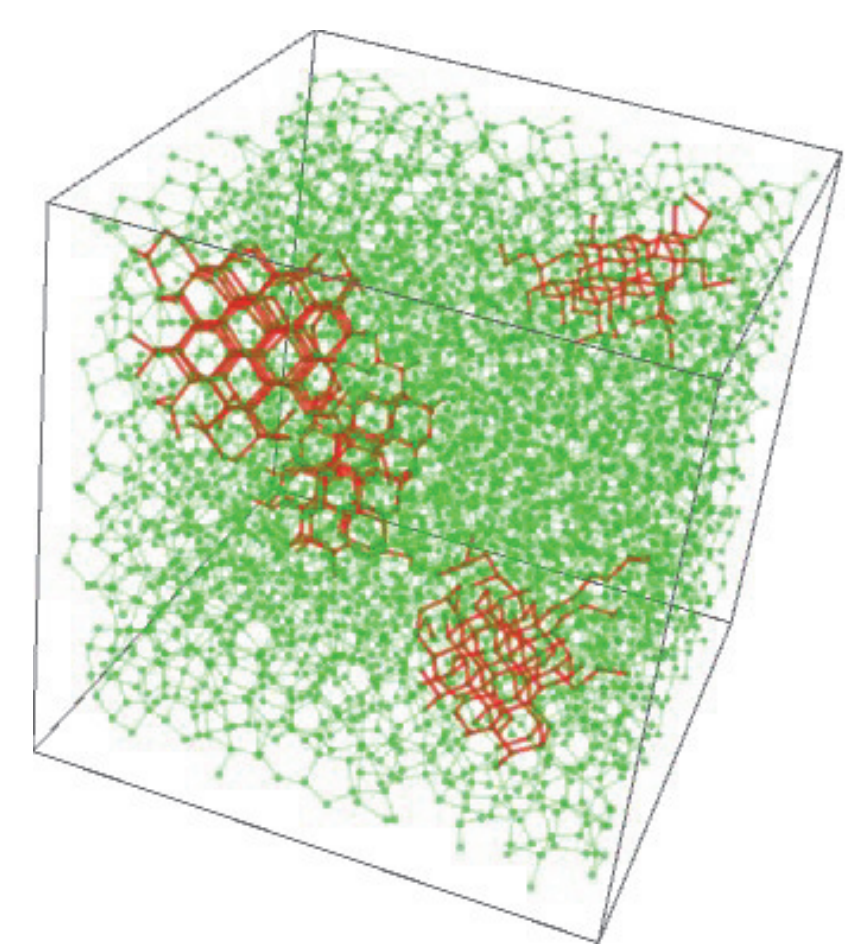

b) Paracrystalline (Nakhmanson)

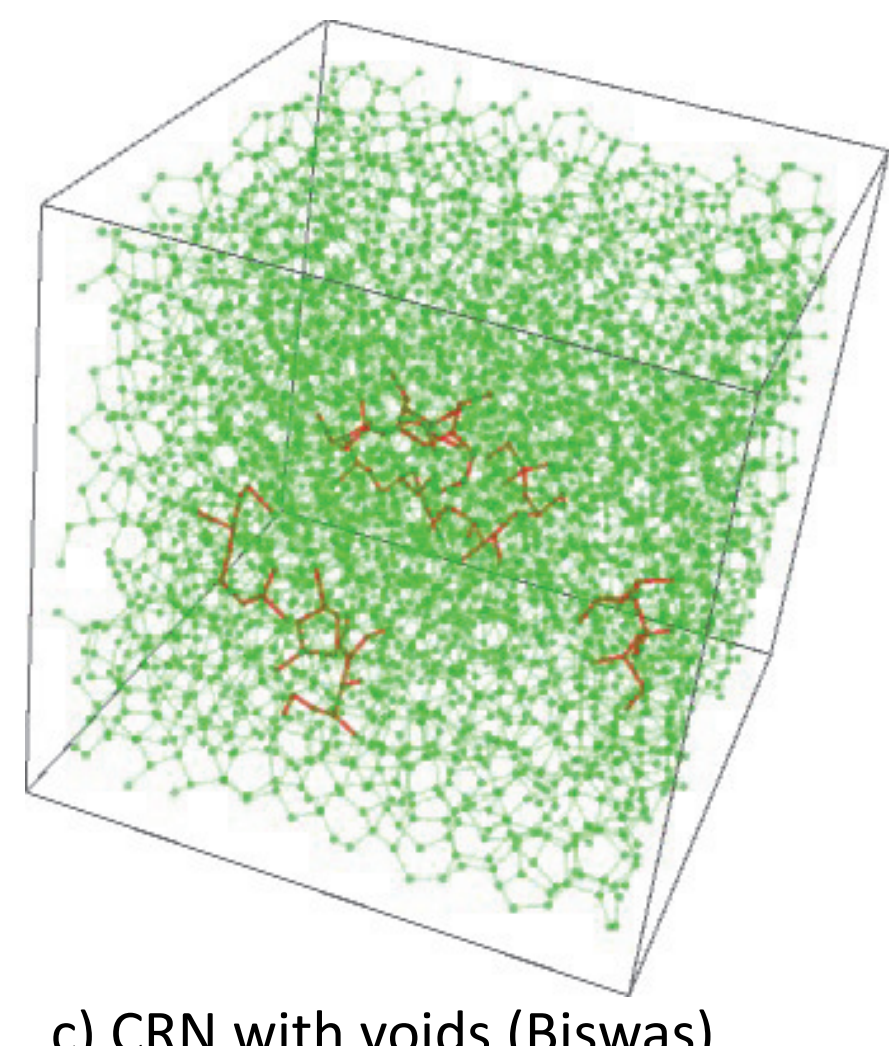

c) CRN with voids (Biswas) 


\section{Figure 2}

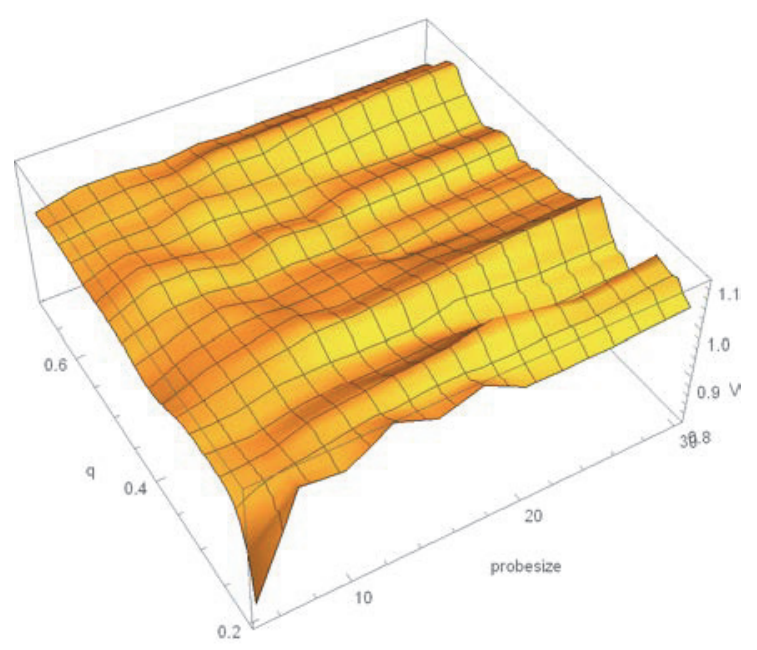

a) CRN (Barkema)
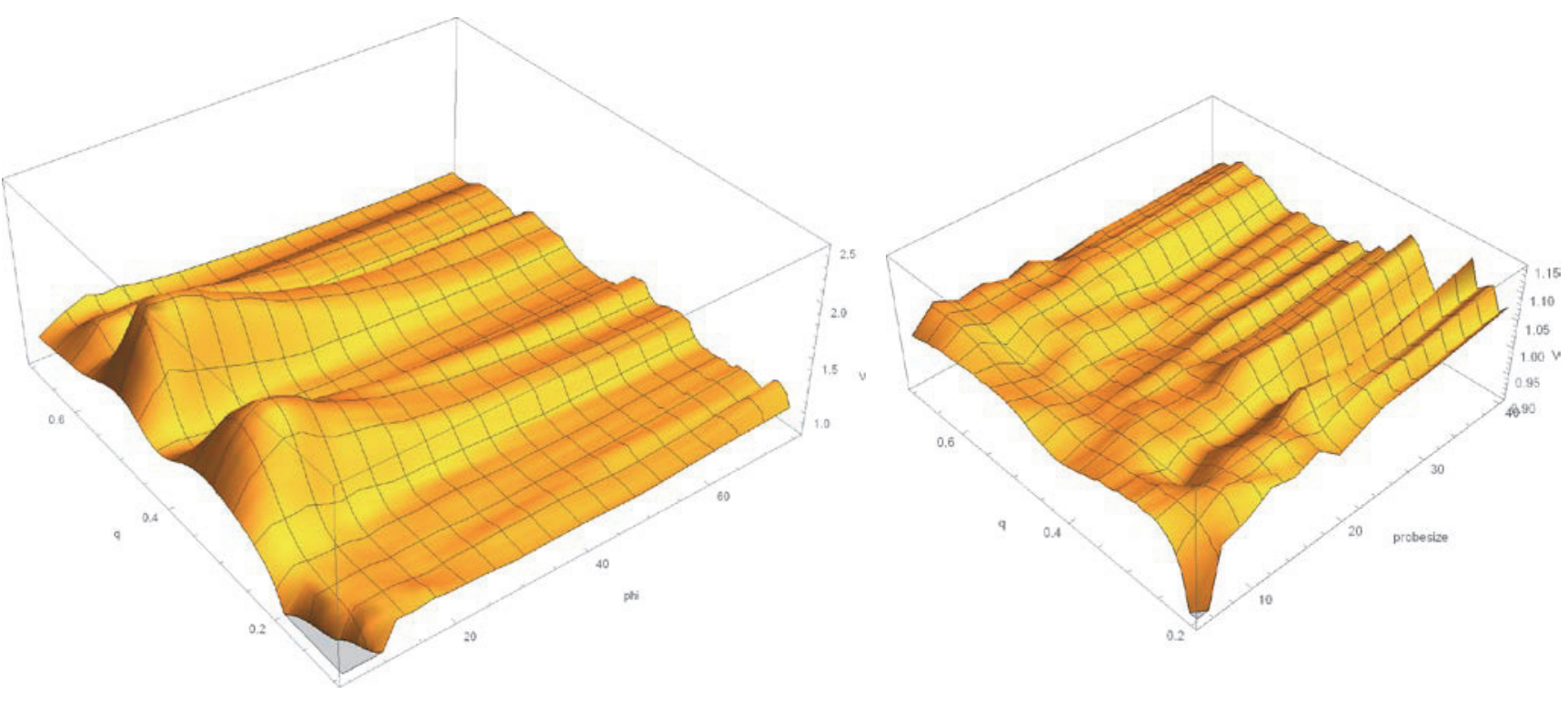

b) Paracrystalline(1.5nm grain size) c) CRN with voids (Biswas) 


\section{Figure 3}

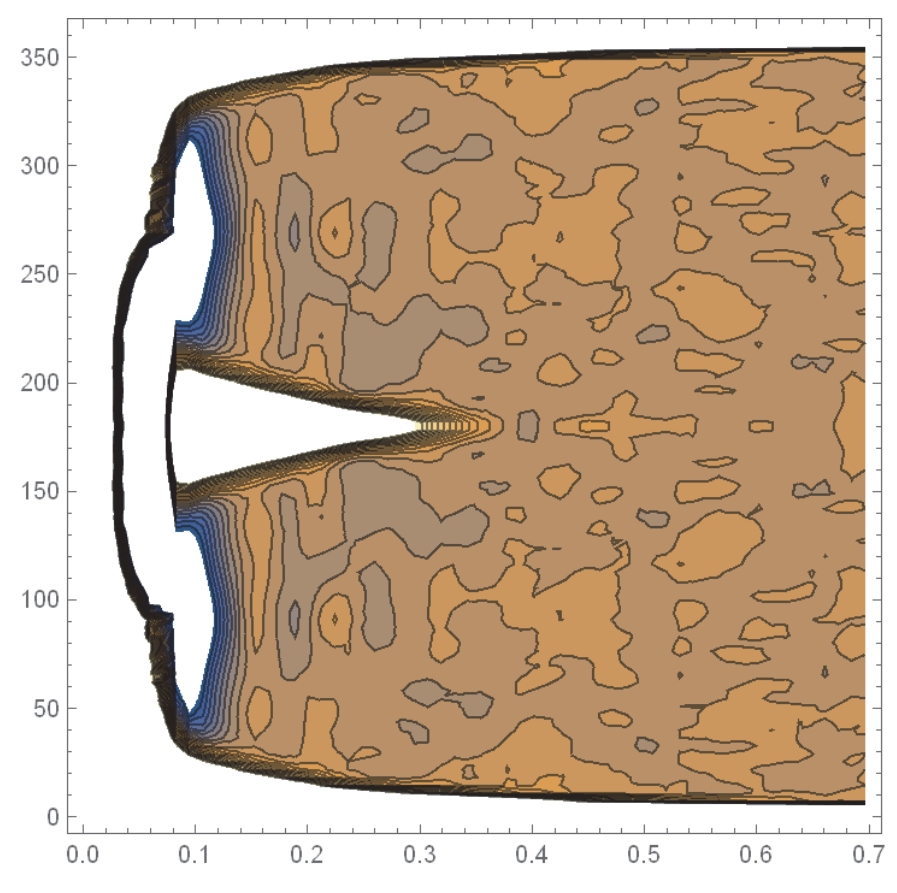

a) CRN Barkema

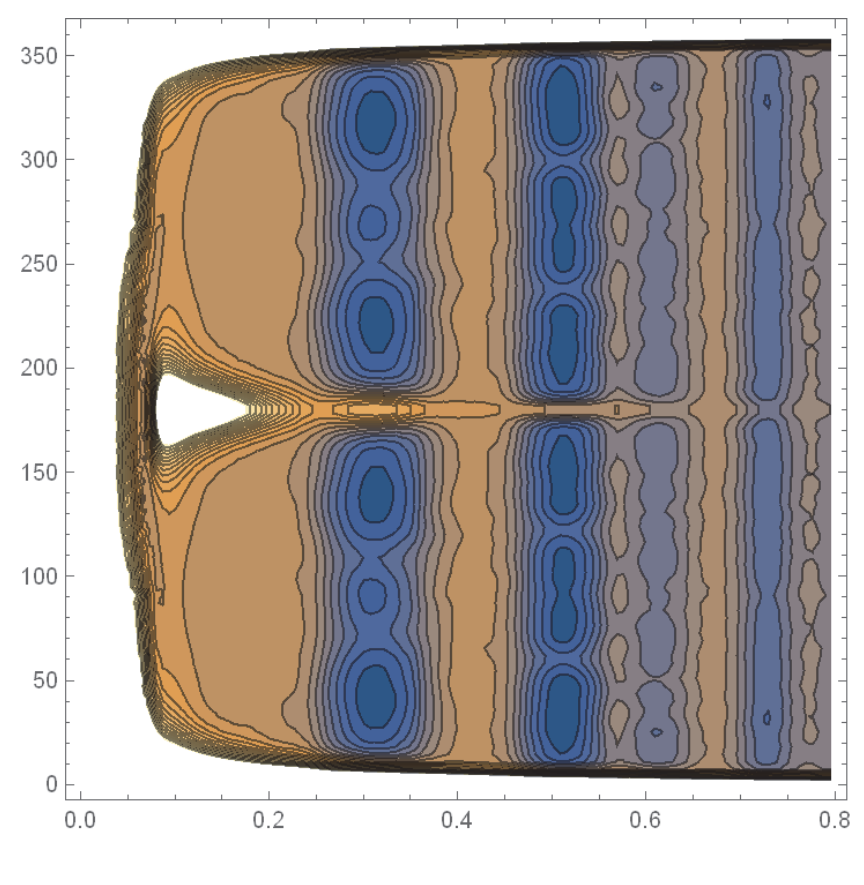

b) Paracrystalline(1.5nm grain size)

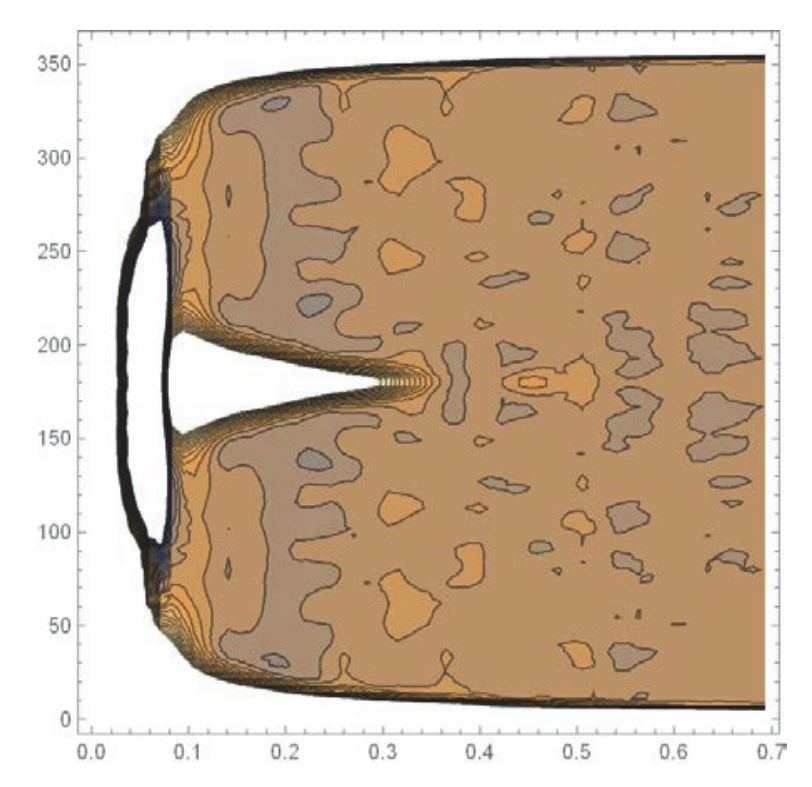

c) CRN with voids (Biswas) 


\section{Figure 4}

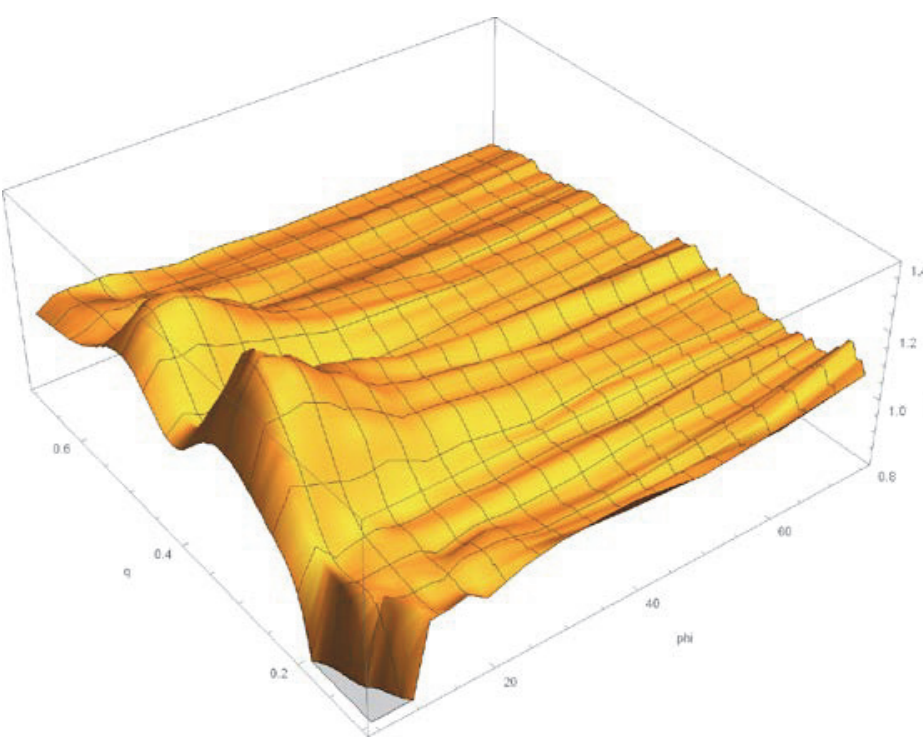

a) $1 \mathrm{~nm}$ grain size

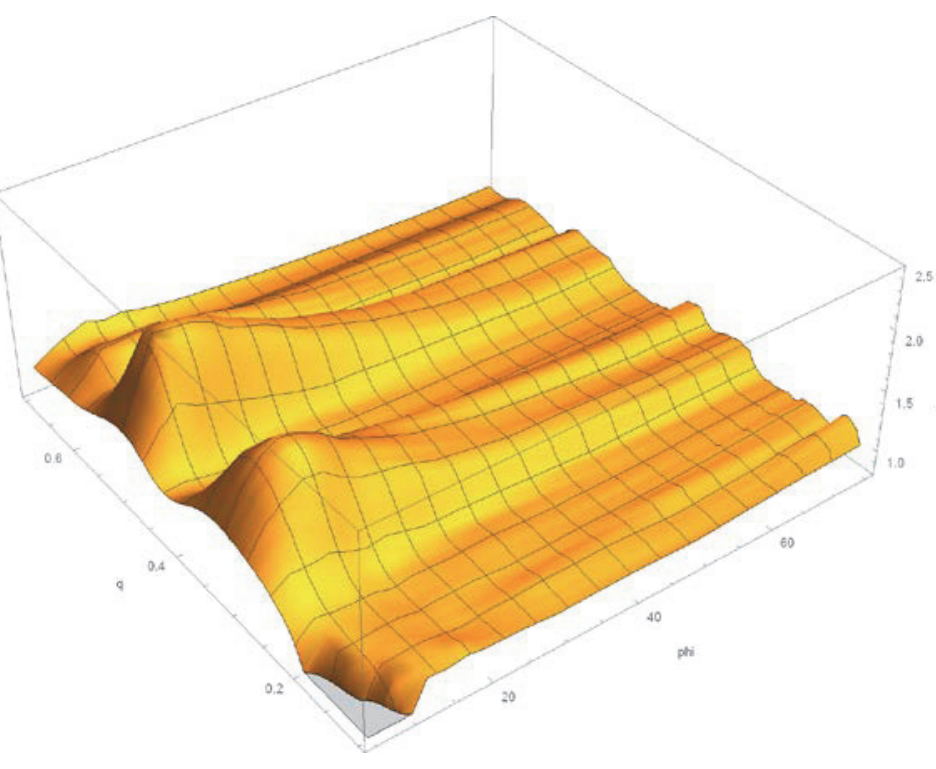

b) $1.5 \mathrm{~nm}$ grain size

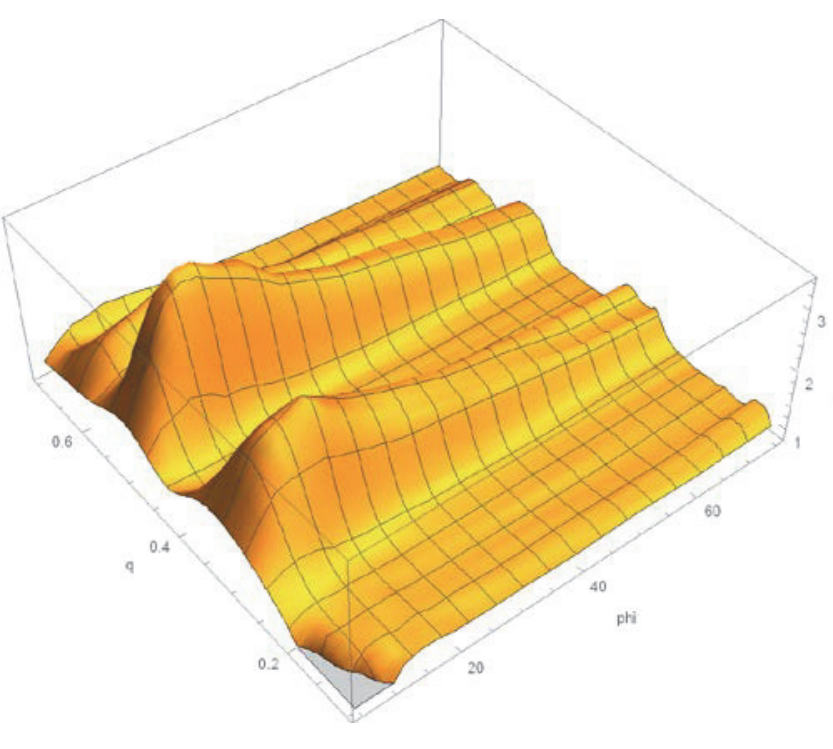

c) $2 \mathrm{~nm}$ grain size 
Figure 5:

d)

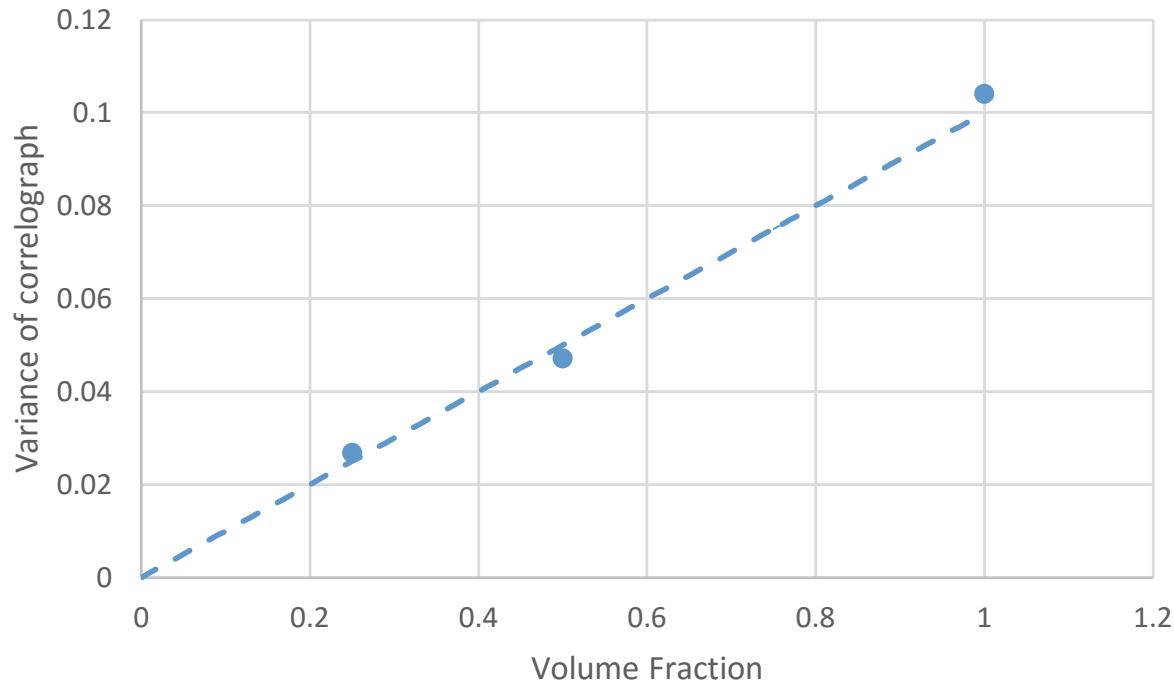

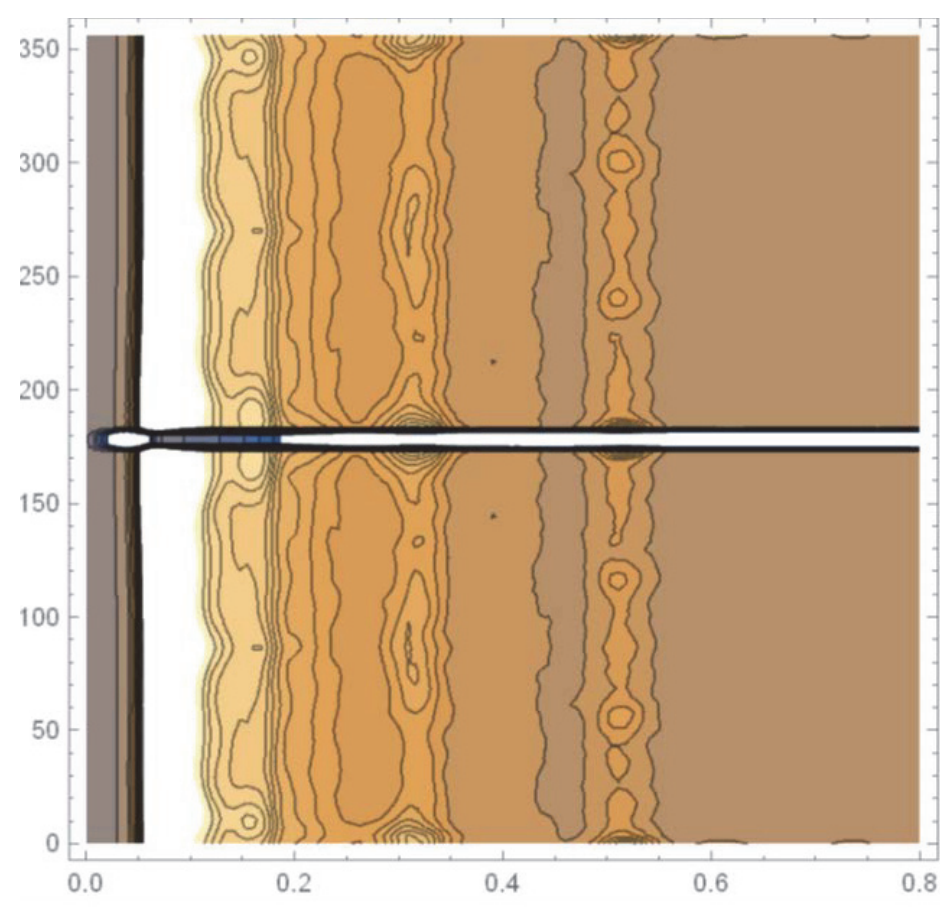

a) $25 \%$

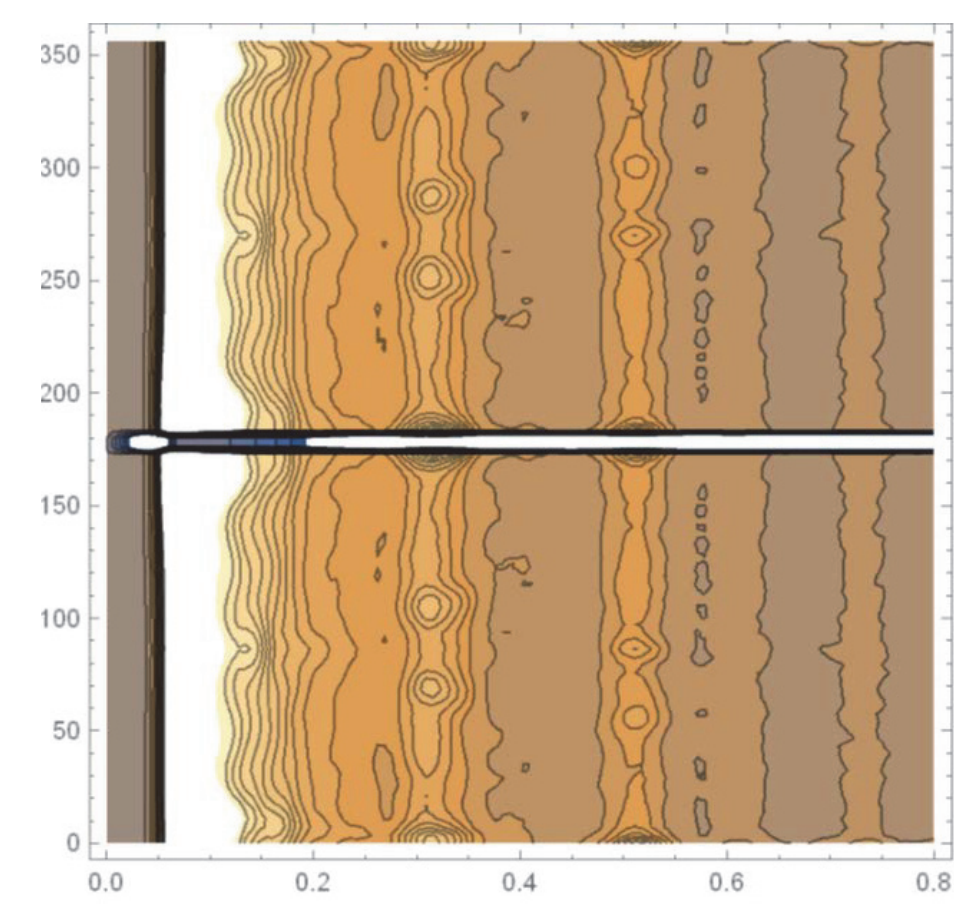

b) $50 \%$

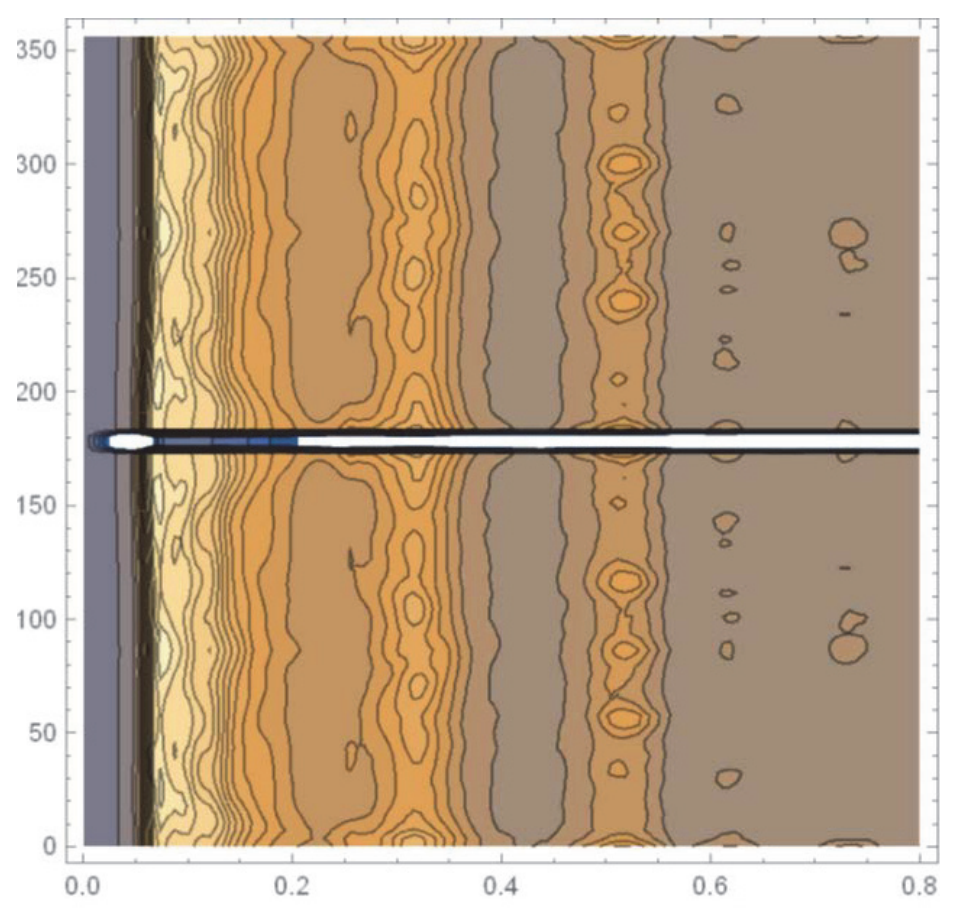

c) $100 \%$ 